\title{
Atlas-Based Automated Segmentation of Spleen and Liver Using Adaptive Enhancement Estimation
}

\author{
Marius George Linguraru, Jesse K. Sandberg, Zhixi Li, John A. Pura, \\ and Ronald M. Summers
}

Imaging Biomarkers and Computer-Aided Diagnosis Laboratory, Radiology and Imaging Sciences, Clinical Center, National Institutes of Health, Bethesda, MD, USA

lingurarum@mail.nih.gov

\begin{abstract}
The paper presents the automated segmentation of spleen and liver from contrast-enhanced CT images of normal and hepato/splenomegaly populations. The method used 4 steps: (i) a mean organ model was registered to the patient CT; (ii) the first estimates of the organs were improved by a geodesic active contour; (iii) the contrast enhancements of liver and spleen were estimated to adjust to patient image characteristics, and an adaptive convolution refined the segmentations; (iv) lastly, a normalized probabilistic atlas corrected for shape and location for the precise computation of each organ's volume and height (mid-hepatic liver height and cephalocaudal spleen height). Results from test data demonstrated the method's ability to accurately segment the spleen $($ RMS error $=1.09 \mathrm{~mm}$; DICE/Tanimoto overlaps $=95.2 / 91)$ and liver $(\mathrm{RMS}$ error $=2.3 \mathrm{~mm}$, and DICE/Tanimoto overlaps $=96.2 / 92.7)$. The correlations $\left(\mathrm{R}^{2}\right)$ with clinical/manual height measurements were 0.97 and 0.93 for the spleen and liver respectively.
\end{abstract}

\section{Introduction}

We are working towards using the fully automated segmentation of the spleen and liver as a volumetric diagnostic tool. It had been noted that the 3D shape and size variability of liver and spleen can be an indication of disorders [3,21]. The implementation of a fully automated segmentation allows the radiologist and other health professionals for an easy and convenient access to organ measurements, while avoiding time-consuming manual measurements or biased diagnosis based on 2D projection images [2]. We propose a method to segment the liver/spleen independent of morphological changes due to disease and/or normal anatomical variability.

In clinical practice, the liver size is estimated by height measurements at the midhepatic line; similarly, the spleen height is measured as the cephalocaudal height. Liver height, for instance, does not fully characterize the morphology of the liver, such as accounting for an enlarged left lobe. Spleen measurements suffer from similar shortcomings. Alternatively, studies have relied on the liver/spleen volume computed by multiplying the calculated slice area from manual segmentations by the slice thickness. [8].

A variety of automated and interactive methods to segment the liver have been proposed. A technique based on statistical analysis and dimensionality reduction from 
sparse information models was presented in [5]. In [4] a shape-guided deformable model was introduced using an evolutionary algorithm, but unacceptable segmentations were omitted in the analysis. Most recently, active contours using gradient vector flow were used to address both liver and hepatic tumor segmentation [12], while a hierarchical statistical atlas was employed in [13]. These methods suffer from either heavy manual initialization or present significant segmentation errors.

In 2007, a liver segmentation competition from computed tomography (CT) data was held [6]. Amongst the automated techniques, most notably a combination of shape-constrained statistical deformable models based on a heuristic intensity model had the best performance amongst automated methods [10] with slight undersegmentation of the liver. Region growing was used in [16] with good results, but the technique was sensitive to liver abnormalities. A semantic formulation of knowledge and context was presented in [17], but the segmentation overlap was only $84 \%$.

Despite the abundance of research on liver segmentation, there are few studies focusing on the spleen. However, the segmentation of abdominal multi-organs, including the liver and spleen, has been addressed, but with limited accuracy. In [19,22] a priori probabilistic data were used in combination with measures of relationship and hierarchy between organs and manual landmarks. On a different note, multidimensional data from contrast-enhanced CT were employed in $[9,11,18]$, applying variational Bayesian mixture and tissue homogeneity constraints.

We propose a method that involves a combination of appearance, enhancement, and shape and location statistics to segment both the spleen and liver. For the coarse estimation of organs, mean models from an atlas of both liver and spleen are aligned to the patient contrast-enhanced CT image. The estimation is improved by a geodesic active contour. Subsequently, the patient specific contrast-enhancement characteristics are estimated and passed to an adaptive convolution. Only homogenous tissue areas that satisfy the enhancement constraints are labeled as liver/spleen. Lastly, shape and location information from the normalized probabilistic atlas are utilized to provide an accurate representation of each organ's morphology.

\section{Methods and Materials}

\subsection{Data and Statistical Information}

192 abdominal CT scans of patients from a mixed population were used; 52 normal livers, 43 normal spleens, 94 splenomegaly (enlarged spleen) and 40 hepatomegaly (enlarged liver) cases. Patients were injected with $130 \mathrm{ml}$ of Isovue-300 and images acquired at portal venous phase using fixed delays or bolus-tracking [7]. Data were collected on LightSpeed Ultra and QX/I (GE Healthcare), and Brilliance64 (Philips Healthcare) scanners. Image resolution ranged from 0.62 to $0.82 \mathrm{~mm}$ in the axial view with a slice thickness from 1 to $5 \mathrm{~mm}$. The livers and spleens were manually segmented in 14 training and 20 testing CT scans, while their heights (mid-hepatic liver height and cephalocaudal spleen height) were manually measured in all data.

For additional tests, we used 20 more contrast-enhanced CT scans with manual segmentations of the liver downloadable from www.sliver07.isi.uu.nl, addressed as MICCAI data in the paper. These CT data were used for the MICCAI 2007 liver 
segmentation competition and were acquired in transversel plane with pixel spacing between 0.55 and $0.80 \mathrm{~mm}$ and inter-slice distance between 1 and $5 \mathrm{~mm}$. Contrastenhanced images corresponded to mainly pathological cases and were acquired on a variety of scanners from different manufacturers.

A probabilistic atlas $A$ was constructed from 10 random CT sets (not included in the training or testing data) after manually segmenting the liver and spleen in each image. Organ locations were normalized to an anatomical landmark (xiphoid). A random image from the set was used as reference $R$ and all other images registered to it. We conserved morphological variability by using a size-preserving affine registration. Restricting the degrees of freedom in the transformation (no shear), the organ shape bias from the reference data is minimized. Preserving the size of organs and normalizing their position to that of the xiphoid, we obtain abdominal location normalization with no bias toward the reference size and location. Finally, organs were translated in the atlas to the location of the average normalized centroid.

\subsection{Registration and Segmentation}

From the construction of $A$, a mean model $\bar{A}$ was extracted for each organ. Then the patient CT $(I)$ was smoothed with anisotropic diffusion [14] and the result is $I_{s}$. First, an affine registration between $R$ and $I$ was perfomed. The resulting spatial normalization was then applied to both $A$ and $\bar{A}$, which became $A_{a}$ and $\bar{A}_{a}$. The affine registration was based on normalized mutual information $M$ [20], where $p\left(I, \bar{A}_{a}\right)$ is the joint probability distribution of images $I$ and $\bar{A}_{a}$, and $p(I)$ and $p\left(\bar{A}_{a}\right)$ their marginal distributions.

$$
M\left(I_{s} \mid \overline{A_{a}}\right)=\frac{p\left(I_{s}\right)+p\left(\overline{A_{a}}\right)}{p\left(I_{s}, \overline{A_{a}}\right)} .
$$

Next, a more flexible registration of $\bar{A}_{a}$ was required to compensate for the residual deformation, resulting in $\overline{A_{r}}$. We employed the non-linear registration algorithm based on B-splines [15]. B-splines allow to locally control the deformation $T$ and a compromise between the similarity provided by $M$ and smoothing $S$ was searched.

$$
\begin{gathered}
\arg \min \left[M\left(I_{s} \mid T\left(I_{s}\right)\right)-S(T)\right] ; \\
S(T)=\int_{x, y, z}\left(\partial^{2} T\right)_{x, y, z} d x d y d z .
\end{gathered}
$$

To account for possibly missing parts of spleen and liver, a geodesic active contour (GAC) [1] was implemented to correct the organ boundaries based on contrastenhanced image intensities. To initialize the model, $\overline{A_{r}}$ was input as zero-level into a GAC $I_{g}$. The edge features $I_{e}$ were computed from the sigmoid of the gradient of $I_{s}$. The weights $w_{1}, w_{2}$ and $w_{3}$ control respectively the speed $c$, curvature $k$ and attraction to edges. In our experimental setup, $w_{1}, w_{2}$ and $w_{3}$ were set to $1,0.2$ and 1 respectively. Parameters $\alpha$ and $\beta$ of the image sigmoid were 10 and 8 respectively. 


$$
\begin{gathered}
I_{e}=1-1 /\left(1+\exp \left[-\frac{\nabla I_{s}-(\alpha+\beta)}{3(\alpha-\beta)}\right]\right) ; \quad I_{g, t=0}=\overline{A_{r}} ; \\
\frac{d I_{g}}{d t}=I_{e}\left(w_{1} c+w_{2} k\right)\left|\nabla I_{g}\right|+w_{3} \nabla I_{e} \nabla I_{g}
\end{gathered}
$$

\subsection{Estimation of Enhancement and Shape Correction}

A common difficulty in processing contrast-enhanced CT data is the estimation of the optimal time for image acquisition. In practice, fixed delays or bolus-tracking techniques [7] are used to approximate the portal venous phase and can yield a different enhancement and appearance of organs. Hence, variations in an organ's enhancement are common and vary between late-arterial and late-portal venous phases. As both the liver and spleen enhance homogeneously at portal venous phase, we estimated the level of enhancement of these organs to reject volumes that were erroneously captured by the GAC. First, the masks of liver and spleen provided by the GAC segmentation are used to computed the mean $\left(\mu_{j}\right)$ and standard deviation $\left(\sigma_{j}\right)$ of the organs $\left(j=1,2\right.$ for liver and spleen). Then outliers are rejected to compute $I_{\max }^{j}=\mu_{j}+2 \sigma_{j}$, and $I_{\min }^{j}=\mu_{j}-2 \sigma_{j}$ to account for each organ enhancement.

$I_{\max }^{j}$ and $I_{\min }^{j}$ are input to an adaptive erosion filter that is applied to $I_{g}$. Thus only regions for which all their voxels in the erosion element $E$ satisfy the intensity criteria are labeled as organs of interest. $L$ represents the labeled image and $l_{j}$ the labels. $L$ is then dilated to account for the convolution with $E$. Finally, the normalized $A_{a}$ (see Section 2.2) is used to correct the shape of the liver/spleen in $L . A_{a}$ resulted from applying an affine transformation (no shear) to the probabilistic atlas constructed with restricted degrees of freedom (preserving the shape). $S$ is the image of the segmented liver and spleen.

$$
L(x, y, z)=\left\{\begin{array}{c}
l_{j}, i f\left(I_{\min }^{j} \leq I_{g} \circ E \leq I_{\max }^{j}\right) ; \quad S=L \cdot A_{a} . \\
0, \text { otherwise }
\end{array}\right.
$$

The volume overlap $(V O)$ of the automatically segmented livers and spleens with the manual segmentations, and Dice coefficient $(D C)$ were calculated.

$$
\begin{gathered}
V O=\frac{V_{\text {manual }} \cap V_{C A D}}{V_{\text {manual }}+V_{C A D}-\left(V_{\text {manaul }} \cap V_{C A D}\right)} ; \\
D C=\frac{2\left(V_{\text {manual }} \cap V_{C A D}\right) .}{V_{\text {manual }}+V_{C A D}} .
\end{gathered}
$$

To correlate with clinical evaluations of liver performed by linear measurements of organ height, the mid-hepatic line (MHL) was approximated at the half-distance between the mid-point of the spine and the outer surface of the liver. Then the maximum liver height along the sagittal plane at the location of MHL was computed. The spleen cephalocaudal height was calculated as the Euclidean distance between the top and bottom sagittal slices containing the spleen. 


\section{Results}

Quantitative results from applying our method to the segmentation of liver and spleen are presented in Table 1. We present DC and VO overlaps next to volume estimation error (VER), height estimation error (HER), root mean square error (RMSE) and average surface distance (ASD). Training cases were of low resolution, while test cases of higher resolution: $5 \mathrm{~mm}$ and $1 \mathrm{~mm}$ slice thickness respectively. We compared results on training data at incremental steps of the algorithm. Results on testing the algorithm on the MICCAI liver data are also provided in Table 1. The segmentation score was 69 , comparable to that of 68 of the competition winner, after using the evaluation tools provided by the competition organizers [6]. Note that our score was obtained on different cases provided by the organizers for training, as we did not have access to the test data used in [6]. Table 1 further presents inter-observer variability for the segmentation of liver and spleen.

Table 1. Statistics for the liver and spleen segmentation results from training and test data. Results at incremental steps of the algorithm are presented for training data: "Atlas"- after nonrigid registration with the probabilistic atlas; 'EE' after enhancement estimation correction; 'Shape' after employing the shape and location correction. Columns present the Dice coefficient (DC), volume overlap (VO), volume estimation error (VER), height estimation error (HER), root mean square error (RMSE) and average surface distance (ASD).

\begin{tabular}{l|cccccc} 
& $\begin{array}{c}\text { DC } \\
(\%)\end{array}$ & $\begin{array}{c}\text { Vo } \\
(\%)\end{array}$ & VER $(\%)$ & HER $(\%)$ & $\begin{array}{c}\text { RMSE } \\
(\mathbf{m m})\end{array}$ & ASD (mm) \\
\hline Training Liver 'Atlas' & $90.9 \pm 3.7$ & $83.6 \pm 6$ & $14.9 \pm 9.6$ & $12.2 \pm 13.2$ & $4.4 \pm 2.1$ & $2.6 \pm 1.2$ \\
Training Liver 'EE' & $94.3 \pm 1.5$ & $89.3 \pm 2.6$ & $3.3 \pm 3.7$ & $3.7 \pm 3.7$ & $3.8 \pm 1.8$ & $1.7 \pm 0.8$ \\
Training Liver 'Shape' & $94.5 \pm 0.8$ & $90 \pm 1$ & $2 \pm 2.1$ & $3.4 \pm 3.1$ & $2.9 \pm 0.5$ & $1.5 \pm 0.3$ \\
\hline Test Liver & $96.2 \pm 0.6$ & $92.7 \pm 1.1$ & $2.2 \pm 2.1$ & $4.5 \pm 6.6$ & $2.3 \pm 0.5$ & $1.2 \pm 0.2$ \\
MICCAI Liver & $95.9 \pm 0.9$ & $92 \pm 1.8$ & $2.9 \pm 2.3$ & $4.3 \pm 4.6$ & $2.9 \pm 1$ & $1.4 \pm 0.5$ \\
\hline Training Spleen 'Atlas' & $87.5 \pm 4.8$ & $78 \pm 7.5$ & $13.6 \pm 10$ & $9.7 \pm 9.2$ & $2.9 \pm 1.2$ & $1.6 \pm 0.7$ \\
Training Spleen 'EE' & $91 \pm 2$ & $83.5 \pm 3.3$ & $6.6 \pm 5.3$ & $3.5 \pm 3.4$ & $2.1 \pm 0.5$ & $1 \pm 0.2$ \\
Training Spleen 'Shape' & $90.6 \pm 2.1$ & $83 \pm 3.5$ & $5.5 \pm 4.9$ & $3.5 \pm 5.1$ & $2.1 \pm 0.6$ & $1.3 \pm 0.8$ \\
\hline Test Spleen & $95.2 \pm 1.4$ & $91 \pm 2.6$ & $3.3 \pm 2.7$ & $1.7 \pm 0.7$ & $1.1 \pm 0.3$ & $0.7 \pm 0.1$ \\
\hline Inter-observer Liver & 96.4 & 92.3 & 1.25 & 3.9 & 1.7 & 0.7 \\
Inter-observer Spleen & 96 & 92.4 & 2.26 & 1.67 & 0.9 & 0.38
\end{tabular}

Figure 1 shows a typical example of liver and spleen segmentation from a test case on 2D axial slices of the 3D CT data. Figure 2 illustrates another example of segmentation in $3 \mathrm{D}$ along with the segmentation errors between manual and automated segmentation. Finally, automated volumetric and linear 3D measurements were obtained for an additional 168 clinical cases: 19 had normal spleens, 29 had normal livers, while 94 had splenomegaly and 40 cases had hepatomegaly. The height correlations $\left(\mathrm{R}^{2}\right)$ between the automatically segmented spleens and livers and the manual linear measurements obtained in clinical practice were 0.97 and 0.93 for spleen and liver respectively, as shown in Figure 3. 


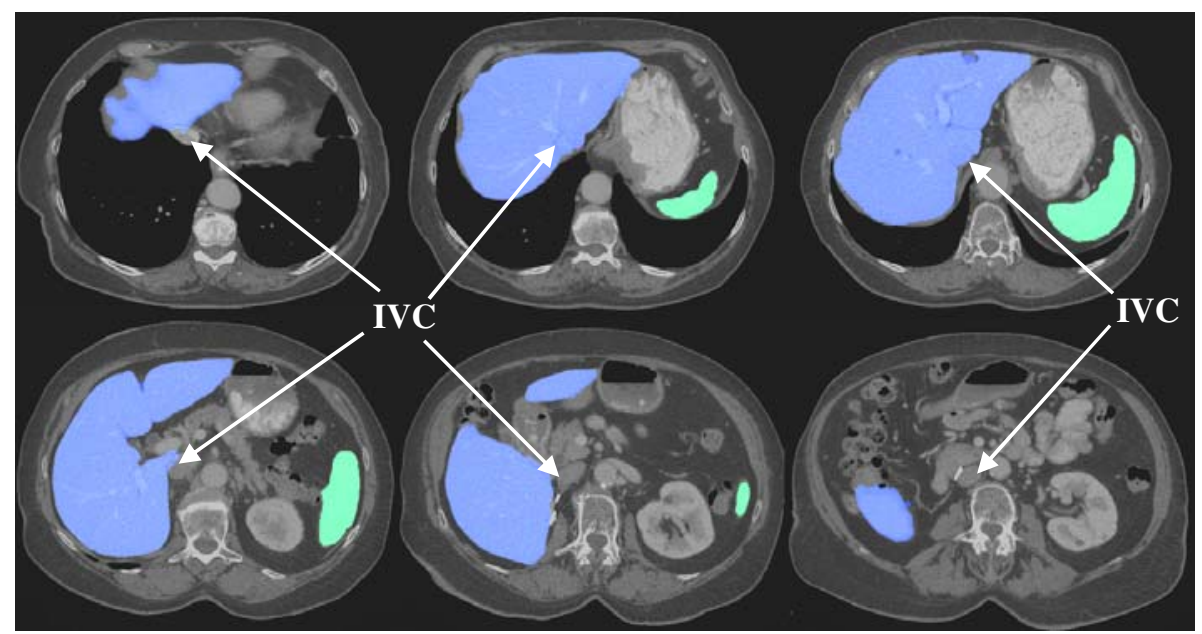

Fig. 1. A typical example of liver (blue) and spleen (green) automatically segmented from a test case on 2D axial views of the 3D CT data. Note the good separation from the heart; parts of the inferior vena cava (IVC) are incorporated in the liver when contrast enhancement is low.

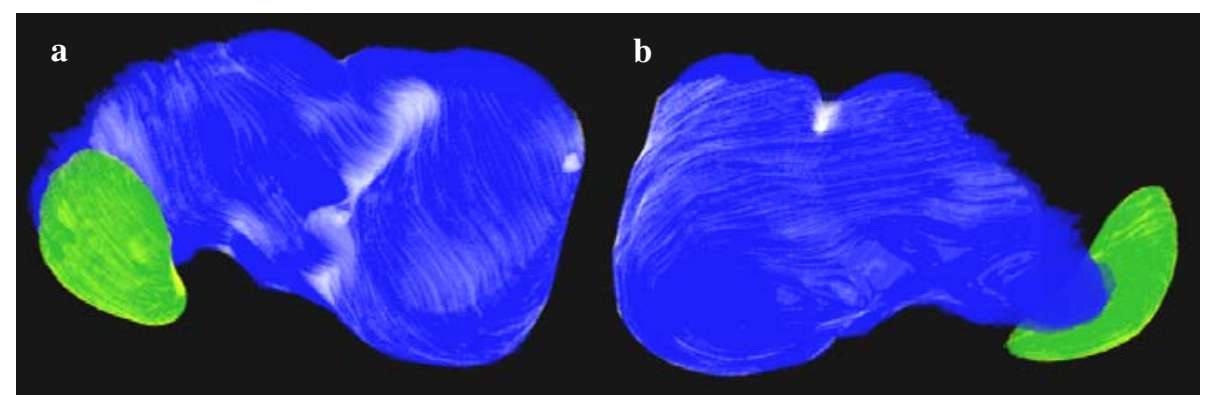

Fig. 2. 3D volume renderings of the segmented liver and spleen; (a) is a posterior view and (b) an anterior view. The liver ground truth is shown in blue with automated segmentation errors overlaid in white; likewise, the spleen ground truth is green and errors are in yellow.
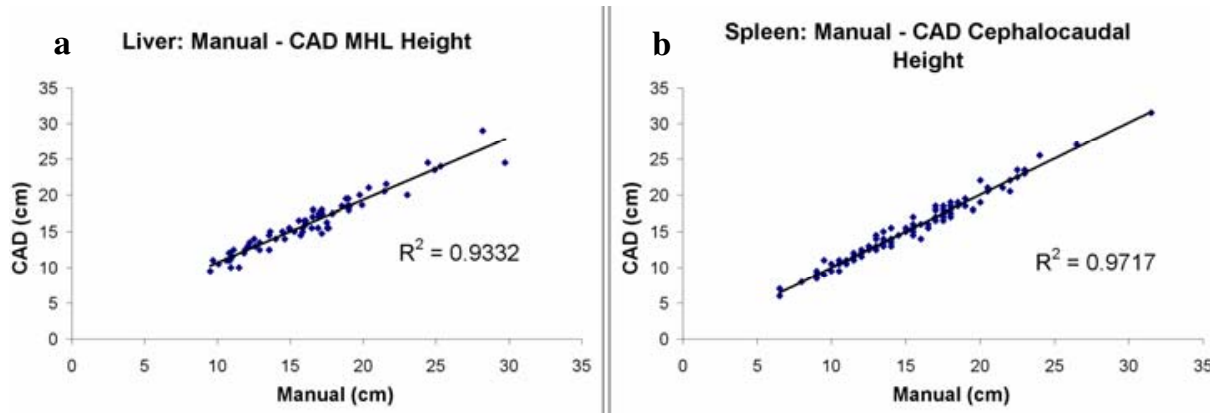

Fig. 3. The correlations $\left(\mathrm{R}^{2}\right)$ between automatically generated (CAD) organ height and the manual measurements obtained in clinical practice; (a) shows correlated liver heights at the mid-hepatic line (MHL) from mixed normal and hepatomegaly cases; (b) presents correlated spleen cephalocaudal heights from mixed normal and splenomegaly cases. 


\section{Discussion}

The proposed method involves a combination of appearance, shape and location statistics to automatically segment livers and spleens from clinical contrast-enhanced CT data of mixed populations with normal and abnormal organs. Data from various institutions and scanners were employed. The patient specific contrast-enhancement characteristics were estimated and input into an adaptive convolution that labeled only homogenous tissue areas that satisfy the enhancement constraints of the liver/spleen. Additionally, shape and location information from the normalized probabilistic atlas were utilized to improve the accuracy of the segmentation. Results demonstrated the ability of the technique to segment normal and abnormal spleens with a precision close to the inter-observer variability and errors close to the voxel size.

The method avoided the inclusion of heart volumes in the segmentation of the liver. Although the inferior vena cava was not incorporated in the liver in the majority of cases, parts of the vein may be errouneously segmented in the mid-cephalocaudal liver region, especially when contrast enhancement was low. Also note that the MICCAI data included pathological cases, which made the segmentation of the liver (including the pathologies) more difficult. As expected, segmentation results were more accurate on data with high spatial resolution.

We found that using shape information from a normalized probabilistic atlas changed/improved significantly $(\mathrm{p}<0.05)$ only the liver volume estimations (see Table 1$)$. This may be explained partly by the small sample of cases used to construct the atlas. But the improvement brought by the adaptive convolution using enhancement estimation and adjusting the parameters to patient specific information was significant for all metrics used in Table $1(\mathrm{p}<0.04)$ in comparison to atlas-based segmentation.

Future work will investigate the use of volumetric measurements to establish more robust diagnosis criteria for the detection of hepato/splenomegaly, and will address additional challenges from a variety of abdominal pathologies. We anticipate to have our method used in routine clinical investigations in the near future.

Acknowledgment. This work was supported by the Intramural Research Program of the National Institutes of Health, Clinical Center.

\section{References}

1. Caselles, V., Kimmel, R., Sapiro, G.: Geodesic active contours. International Journal on Computer Vision 22(1), 61-97 (1997)

2. Cools, L., Osteaux, M., Divano, J.L.: Prediction of Splenic volume by a Simple CT Measurement: A Statistical Study. J. Comput. Assist. Tomogr. 7(3), 426-430 (1983)

3. Ellert, J., Kreel, L.: The role of computed tomography in the initial staging and subsequent management of the lymphomas. J. Comput. Assist. Tomogr. 4, 358 (1980)

4. Farraher, S.W., Jara, H., Chang, K.J., Hou, A., Soto, J.A.: Liver and Spleen Volumetry with Quantitative MT Imaging and Dual-Space Clustering Segmentation. Radiology 237(1), 322-328 (2005)

5. Florin, C., Paragios, N., Funka-Lea, G., Williams, J.: Liver segmentation using sparse 3D prior models with optimal data support. Information Processing in Medical Imaging 20, 38-49 (2007) 
6. van Ginneken, B., Heimann, T., Styner, M.: 3D Segmentation in the Clinic: A Grand Challenge. In: van Ginneken, B., Heimann, T., Styner, M. (eds.) 3D Segmentation in the Clinic: A Grand Challenge, pp. 7-15 (2007)

7. Goshima, S., Kanematsu, M., Nishibori, H., Kondo, H., Tsuge, Y., Yokoyama, R., Miyoshi, T., Onozuka, M., Shiratori, Y., Moriyama, N., Bae, K.T.: Multi-detector row CT of the kidney: optimizing scan delays for bolus tracking techniques of arterial, corticomedullary, and nephrographic phases. European Journal of Radiology 63(3), 420-426 (2007)

8. Heymsfield, S.B., Fulenwider, T., Nordlinger, B., Barlow, R., Sones, P., Kutner, M.: Accurate measurement of liver, kidney, and spleen volume and mass by computerized axial tomography. Ann. Intern. Med. 90, 185-187 (1979)

9. Hu, X., Shimizu, A., Kobatake, H., Nawano, S.: Independent component analysis of fourphase abdominal CT images. In: Barillot, C., Haynor, D.R., Hellier, P. (eds.) MICCAI 2004. LNCS, vol. 3217, pp. 916-924. Springer, Heidelberg (2004)

10. Kainmueller, D., Lange, T., Lamecker, H.: Shape Constrained Automatic Segmentation of the Liver based on a Heuristic Intensity Model. In: van Ginneken, B., Heimann, T., Styner, M. (eds.) 3D Segmentation in the Clinic: A Grand Challenge, pp. 109-116 (2007)

11. Linguraru, M.G., Summers, R.M.: Multi-Organ Segmentation in 4D Contrast-Enhanced Abdominal CT. In: IEEE Symposium on Biomedical Imaging 2008 (ISBI), pp. 45-48 (2008)

12. Massoptier, L., Casciaro, S.: A New Fully Automatic and Robust Algorithm for Fast Segmentation of Liver Tissue and Tumors from CT Scans. European Radiology 18, 1658-1665 (2008)

13. Okada, T., Shimada, R., Hori, M., Nakamoto, M., Chen, Y.W., Nakamura, H., Sato, Y.: Automated Segmentation of the Liver from 3D CT Images Using Probabilistic Atlas and Multilevel Statistical Shape Model. Academic Radiology 15, 1390-1403 (2008)

14. Perona, P., Malik, J.: Scale-space and Edge Detection using Anisotropic Diffusion. IEEE Trans. on Pattern Analysis and Machine Intelligence (12), 629-639 (1990)

15. Rueckert, D., et al.: Non-rigid registration using free-form deformations: Application to breast MR images. IEEE Transactions on Medical Imaging 18(8), 712-721 (1999)

16. Rusko, L., Bekes, G., Nemeth, G., Fidrich, M.: Fully automatic liver segmentation for contrast-enhanced CT images. In: van Ginneken, B., Heimann, T., Styner, M. (eds.) 3D Segmentation in the Clinic: A Grand Challenge, pp. 143-150 (2007)

17. Schmidt, G., Athelogou, M., Schoenmeyer, R., Korn, R., Binnig, G.: Cognition Network Technology for a Fully Automated 3D Segmentation of Liver. In: van Ginneken, et al. (eds.) 3D Segmentation in the Clinic: A Grand Challenge, pp. 125-133 (2007)

18. Sakashita, M., Kitasaka, T., Mori, K., Suenaga, Y., Nawano, S.: A Method for Extracting Multi-organ from Four-phase Contrasted CT Images based on CT Value Distribution Estimation using EM-algorithm. In: Proceedings of SPIE, vol. 6509, 1C-1-12 (2007)

19. Shimizu, A., Ohno, T., Ikegami, R., Kobatake, H.: Multi-organ Segmentation in Threedimensional Abdominal CT Images. Int. J. CARS 1, 76-78 (2006)

20. Studholme, C., Hill, D.L.G., Hawkes, D.J.: An overlap invariant entropy measure of 3D medical image alignment. Pattern Recognition 32(1), 71-86 (1999)

21. Tsushima, Y., Endo, K.: Spleen Enlargement in Patients with Nonalcoholic Fatty Liver Correlation between Degree of Fatty Infiltration in Liver and Size of Spleen. Digestive Diseases and Sciences 45(1), 196-200 (2000)

22. Yao, C., Wada, T., Shimizu, A., Kobatake, H., Nawano, S.: Simultaneous Location Detection of Multi-organ by Atlas-guided Eigen-organ Method in Volumetric Medical Images. Int. J. CARS 1, 42-45 (2006) 